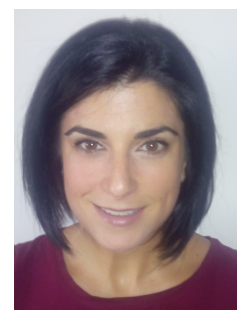

\title{
La gestión de la terminología en interpretación social a través de dispositivos móviles
}

María Teresa Ortego Antón Universidad de Valladolid Departamento de Lengua Española Área de Traducción e Interpretación

\section{RESUMEN}

Los traductores tienen a su disposición una gran cantidad de herramientas para gestionar la terminología, pero los intérpretes sociales no se han beneficiado del mismo nivel de innovación y su metodo de trabajo todavía es rudimentario. En consecuencia, basándonos en la literatura precedente, en este trabajo establecemos una serie de parámetros para evaluar varias herramientas de gestión terminológica desde la perspectiva de los intérpretes sociales. Con los resultados que obtengamos extraeremos las conclusiones, que señalarán la necesidad de tener en cuenta nuevas propuestas para implementar las herramientas de gestión terminológica para la interpretación social así como de ofrecer nuevos procedimientos de búsqueda a intérpretes sociales tanto profesionales como en formación.

Palabras clave: gestión terminológica, interpretación social, herramientas de traducción asistida por ordenador, evaluación, parámetros

\section{ABSTRACT}

Translators have a great variety of tools and applications for terminology management. However, social interpreters have not benefited from the same level of innovation and their working methods are still rudimentary. Consequently, in this paper we establish a set of parameters from the review of literature to assess to what extent terminology management tools meet social interpreters' needs. Then, a selection of terminology management tools is analysed. From the results, conclusions are drawn which might suggest a need to take into account new proposals in order to implement terminology management in computerassisted tools from the view of community interpreting as well as to inform new searching procedures used by trainees and community interpreters.

Keywords: terminology management, community interpreting, computer-assisted tools, assessment, parameters

\section{RESUM}

Els traductors tenen a l'abast una gran quantitat d'eines i d'aplicacions per a gestionar la terminologia. Tanmateix, els intèrprets socials, la funció dels quals és permetre a la població que no domina la llengua oficial del país accedir als serveis bàsics i comunicarse amb els proveïdors d'aquests serveis (Shlessinger, 2011: 6), no s'han beneficiat del mateix nivell d'innovació, i la seva metodologia de treball continua essent rudimentària i manual. En conseqüència, en aquest treball pretenem establir una sèrie de paràmetres basant-nos en la bibliografia precedent per a avaluar diverses eines de gestió terminològica tenint en compte les necessitats dels intèrprets socials. Amb els resultats que n'obtinguem, extraurem les conclusions, que apuntaran la necessitat de tenir en compte noves propostes per implementar les eines de gestió terminològica per a la 
interpretació social, com també d'oferir nous procediments de cerca a intèrprets socials professionals i en formació.

Paraules clau: gestió terminològica, eines de traducció assistida per ordinador, interpretació social, avaluació, paràmetres

\section{La interpretación social y los dispositivos móviles}

En la última década han aparecido en el mercado nuevos dispositivos, por ejemplo teléfonos móviles, tabletas y relojes, con funciones semejantes a los ordenadores que, además, disponen de conexión a Internet. Conscientes de estas transformaciones, la industria de la traducción asistida por ordenador (TAO de ahora en adelante) ha creado infinidad de aplicaciones y de programas para facilitar y asistir a los traductores e intérpretes durante la transferencia interlingüística.

Por lo que respecta a la gestión terminológica, la atención se ha focalizado sobretodo en los traductores. Sin embargo, no se ha prestado la suficiente atención a las necesidades terminológicas de los intérpretes, a pesar de que trabajan en campos del saber con un alto grado de especialidad.

Por otro lado, dentro de los distintos tipos de interpretación, la interpretación social, cuya finalidad es permitir a los usuarios de servicios públicos que no tienen competencia en las lenguas oficiales del país comunicarse con los proveedores de los mencionados servicios (Roberts, 1994: 127), comenzó a estudiarse a partir de 1970, aunque la norma ISO relativa a este tipo de interpretación no fue aprobada hasta 2014. Prueba de esta juventud, como señalan varios autores (Mikkelson, 2004; Pöchhaker, 2011; Bancroft, 2015: 218; Tipton y Furmanek, 2016: 3-5), es la coexistencia de varias denominaciones para describir esta actividad: interpretación en los servicios públicos en Reino Unido, interpretacion social en Austria, Canadá, Portugal o Estados Unidos, interpretación en los servicios públicos e interpretación social en España o mediación lingüística y cultural en Alemania. En este trabajo optamos por utilizar la denominación interpretación social, puesto que consideramos que esta actividad no se desempeña exclusivamente en los servicios públicos, sino que también incluye aquellas situaciones en la que los individuos o grupos de una sociedad que no dominan la lengua oficial intentan acceder a servicios básicos y necesitan intermediarios para comunicarse con los proveedores de los mencionados servicios (Schlesinger, 2011: 6). De hecho, los intérpretes sociales son intermediarios entre dos lenguas y dos culturas, por lo que se ven obligados a coordinar, mediar y negociar el contenido lingüístico, social y cultural (Valero Garcés, 2014: 27).

Dado que una de las competencias básicas que los intérpretes sociales tienen que dominar se corresponde con la gestión terminológica (Tipton y Furmanek, 2016: 6), los dispositivos móviles se han convertido en herramientas muy útiles para almacenar, buscar y recuperar la información terminológica. No obstante, para evitar conflictos durante la interpretación, conviene explicar sucintamente su función tanto al usuario como al proveedor de los servicios de interpretación social. No obstante, el intérprete social presta sus servicios en distintos ámbitos como el médico, el jurídico, el educativo o los servicios sociales (Bancroft, 2015: 221), de manera que la gestión de la terminología tiene un papel crucialy puede verse simplfiicada con el empleo de las herramientas en los dispositivos móviles.

En consecuencia, en el presente trabajo pretendemos establecer una serie de parámetros que utilizaremos para analizar una selección de herramientas de gestión terminológica disponibles para dispositivos móviles en el mercado así como comprobar en qué medida satisfacen las necesidades de los intérpretes sociales. 


\section{Terminología e interpretación}

\subsection{Introducción}

Aunque son muchos los trabajos en los que se estudia la terminología desde la perspectiva del traductor, la realidad es que han sido pocos los que se centran en la terminología desde el punto de vista del intérprete (Gile, 1985; 1986; 1987; Moser-Mercer, 1992; Rodríguez y Snell, 2009; Bilgen, 2009; Costa et al., 2014a; 2014b; 2015). De hecho, en estos estudios se pone de relevancia que las herramientas dirigidas a este colectivo son insuficientes e incapaces de satisfacer todos los requisitos (Costa et al., 2014a). Además, están orientadas principalmente a la interpretación de conferencias (Costa et al., 2015).

Tradicionalmente el método de trabajo de los intérpretes solía ser manual, pero con la introducción de las herramientas TAO y el uso de Internet y la introducción de herramientas TAO la preparación de las interpretaciones se ha transformado, distinguiéndose cuatro fases (Kutz, 2000):

1. La preparación del tema.

2. La preparación lingüística.

3. La preparación de la traducción.

4. La preparación de la interpretación.

Durante la primera fase, el intérprete se familiariza con el campo del saber, busca información, define los campos de la base de datos y extrae los términos. A continuación, analiza el material que ha compilado y recupera la terminología específica del dominio, así como los sinónimos, los hiperónimos, los acrónimos y otras formas abreviadas. En la tercera fase, el intérprete transfiere los términos a la lengua meta utilizando los datos extraídos del corpus en las lenguas de trabajo. Por último, elimina redundancias. Además, tras la interpretación, la base de datos que ha elaborado no es un producto cerrado, sino que realiza correcciones y adiciones para evitar la pérdida de información.

\subsection{La gestión terminológica en interpretación social}

Una vez descrito el método de trabajo, el intérprete social, por las características de este tipo de interpretación, tiene que haber gestionado la terminología antes de realizar el trasvase interlingüístico. En consecuencia, surge la cuestión del tipo de información que necesita incluir en el registro de los términos. Algunos campos serán idénticos a los necesarios en traducción, por ejemplo, cualquier entrada en una base de datos terminológica necesita el campo, el subcampo, el término en la lengua origen y el equivalente en la lengua meta, la definición y un ejemplo. No obstante, el intérprete social tiene que prever que el usuario podría desconocer los términos en su propia lengua por tratarse de un campo de especialidad que no domina, por lo que se verá obligado a incluir traducciones monodireccionales de los términos técnicos, así como información contextual y equivalentes exactos o funcioanles (Hebenstreit y Soukup-Unterweger, 2011). También debería incluir hiperónimos y sinónimos, así como información contextual del término, abreviaciones, nombres propios o nombres de producto, información relativa al registro y a las preferencias del proveedor de servicios, la pronunciación y las unidades fraseológicas o las colocaciones del tipo verbo-nombre que le facilitarán la reconstrucción del mensaje.

Por lo que respecta al formato visual de las fichas, es necesario que el intérprete localice la información de un solo vistazo, por lo que es esencial considerar la posibilidad de desarrollar bases de datos de pequeño tamaño en función del campo del saber o según la situación y el proveedor de servicios. Además, este tipo de bases deberían ser multilingües y permitir al intérprete cambiar entre la lengua origen y la lengua meta. 
En relación con los requisitos del software, Rodríguez y Snell (2009: 27) distinguen cinco características que las herramientas terminológicas destinadas a la interpretación social deberían incluir:

1. Velocidad de consulta rápida.

2. Navegación intuitiva.

3. Posibilidad de actualizar la ficha durante la interpretación o tras la misma.

4. Libertad para definir la estructura básica.

5. Diferentes formas de filtrado de datos según las necesidades del intérprete.

Aunque algunos intérpretes todavía guardan la información en papel o en tablas de Excel, actualmente se han desarrollado herramientas específicamente diseñadas para buscar, guardar y gestionar la terminología, que pueden clasificarse según sean independientes o se integren en un paquete de herramientas TAO (Bilgen, 2009: 28-35).

\section{Metodología de análisis}

\subsection{Herramientas de gestión terminológica para intérpretes}

En primer lugar, es necesario definir las herramientas que vamos a evaluar, puesto que en el mercado existe una gran variedad. Por tanto, en este trabajo hemos limitado el análisis a las herramientas más utilizadas por la International Association of Conference Interpreters (Rütten, 2014a, 2014b). Nos hubiese gustado utilizar los resultados aplicados a la interpretación social, pero actualmente todavía no existe una asociación internacional.

A continuación, ofrecemos una breve descripción de las herramientas:

- Glossary Assistant es una herramienta intuitiva disponible en Google Play con múltiples alfabetos, permite la creación de glosarios de gran tamaño y la capacidad se limita a la memoria disponible en el dispositivo. Además, se pueden importar glosarios existentes en otros formatos. Se basa en el toque con el dedo y la interfaz y el sistema de búsqueda son muy intuitivos.

- Interplex es un programa de gestión de glosarios multilingüe para traductores e intérpretes que puede utilizarse de forma fácil y rápida mientras se interpreta. Se pueden importar glosarios en otros formatos y el usuario puede buscar en todos ellos o seleccionar la búsqueda en uno determinado, así como editarlos. Sin embargo, no permite clasificar y filtrar los términos en función del cliente, del campo, del proyecto ni de la fecha. También está disponible para dispositivos iOS, pero la versión es más simple y se denomina Interplex Lite para iPhone e Interplex HD para iPad.

- InterpretBank es una herramienta de gestión terminológica diseñada para intérpretes. Permite compartir glosarios con colegas, sincronizarse en varios dispositivos, tiene funciones de importación y exportación, y traducción automática. Además, permite buscar en glosarios mientras se trabaja y es una herramienta de fácil uso para memorizar términos. Asiste al intérprete a prepararse para un nuevo proyecto de interpretación, le permite interactuar con la web para buscar traducciones y definiciones o términos en los textos de referencia del intérprete.

- Interpreters Help es una aplicación basada en la web que funciona en cualquier dispositivo. Se organiza en glosarios con funciones de edición, un número ilimitado de lenguas pero sí que limita el número de campos: comentario, categoría, definición, acrónimo y otras. Permite al usuario actualizar la terminología en todos los dispositivos: la nube, el ordenador, la tableta y el teléfono móvil. También permite compartir la información con los colegas. La función de búsqueda es muy rápida, con una lista que se va acotando según se van incluyendo más caracteres. 
- Lookup es una herramienta basada en la web con una rápida función de búsqueda: cuando el usuario introduce las primeras letras, aparecen los resultados. Se limita a cuatro lenguas y solo ofrece tres al mismo tiempo. Tiene muchos campos: cliente, campo, proyecto, etc., e incluso ofrece las relaciones semánticas.

- Terminus es un sistema de gestión terminológica multilingüe diseñado por intérpretes para intérpretes. Entre sus características, destaca la rapidez de las búsquedas, la capacidad de importación y exportación a otros formatos y la posibilidad de clasificar los datos en función de los glosarios, al empresa o los grupos. Además, las búsquedas pueden limitarse a un determinado campo del saber.

\subsection{Parámetros de análisis}

Teniendo en cuenta las necesidades de los intérpretes sociales, hemos establecido los parámetros para analizar las herramientas previamente descritas basándonos en trabajos precedentes (Bilgen, 2009; Rodríguez \& Snell, 2009; Costa et al. 2014a, 2014b, 2015).

En primer lugar, hemos clasificado los parámetros en cuatro grupos en función de las características, es decir, si hacen referencia al software, si están relacionados con la facilidad de uso de la herramienta, con el motor de búsqueda o con el diseño de la base de datos.

Por lo que respecta a las características del software, comprobamos si la herramienta incluye a los intérpretes entre sus usuarios potenciales y si son sistemas autónomos o tienen que utilizarse dentro de un paquete de herramientas TAO. El precio y la posibilidad de uso sin Internet también la valoramos, puesto que en ocasiones los intérpretes sociales tienen que trabajar en lugares públicos donde no hay conexión a internet. Las combinaciones lingüísticas es otra característica que tenemos en cuenta en este bloque, puesto que deberían ofrecer cobertura de un amplio abanico de lenguas. Asimismo, comprobaremos si se pueden emplear distintos sistemas de escritura, por ejemplo para lenguas como el ruso o el árabe. El último parámetro es el relativo al sistema operativo, puesto que deberían funcionar con Windows, iOS y Android para no limitar el dispositivo.

La segunda sección está relacionada con la facilidad de uso, es decir, tiene que ser fáciles de usar puesto que los intérpretes sociales no tienen mucho tiempo para aprender cómo utilizar una nueva herramienta, dado que su esfuerzo tiene que ir encaminado a preparar la interpretación.

En relación a la tercera sección, la función de búsqueda, por un lado analizamos si el motor de búsqueda es rápido y si permite filtrar datos según diferentes necesidades.

La última sección se corresponde con la base de datos. Valoramos si se puede definir la estructura según las necesidades de cada proyecto, si permite actualizar la información y si se puede exportar e importar la información a otros formatos como, por ejemplo, TXT, PDF, XLSX, XML y TMX.

\section{Análisis y resultados}

Para presentar los resultados hemos elaborado una tabla con los parámetros y hemos comprobado si las herramientas seleccionadas los cumplen. 


\begin{tabular}{|c|c|c|c|c|c|c|c|c|c|c|c|c|c|}
\hline \multirow[b]{2}{*}{ 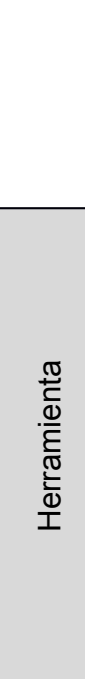 } & \multicolumn{7}{|c|}{ Requisitos del software } & $\stackrel{\circ}{\circ}$ & \multicolumn{2}{|c|}{ 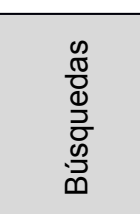 } & \multicolumn{3}{|c|}{$\begin{array}{l}0 \\
0 \\
0 \\
0 \\
0 \\
0 \\
0 \\
0 \\
0 \\
0 \\
\infty\end{array}$} \\
\hline & 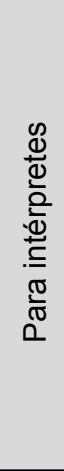 & 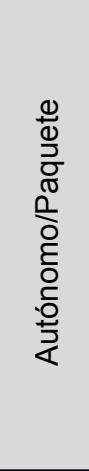 & $\begin{array}{l}\frac{0}{0} \\
\frac{0}{0} \\
\end{array}$ & 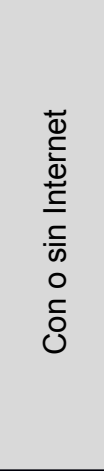 & 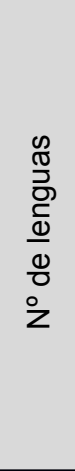 & 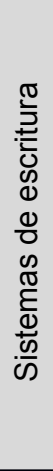 & 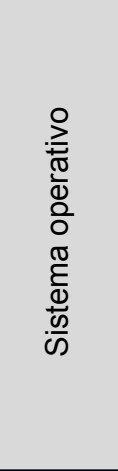 & 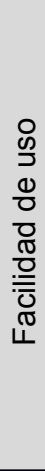 & 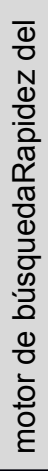 & 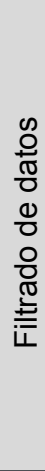 & 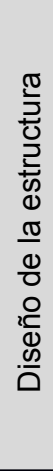 & 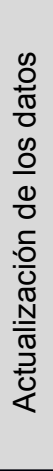 & 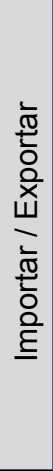 \\
\hline 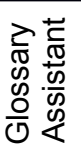 & Sí & A & Gratis & $\operatorname{Sin}$ & llim. & & $\begin{array}{c}\text { Android } \\
\text { Win }\end{array}$ & Sí & Sí & No & Sí & Sí & Sí \\
\hline 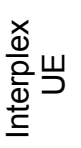 & Sí & A & $\$ 75$ & Sin & 180 & No & $\begin{array}{c}\text { Android } \\
\text { Win } \\
\text { iOS }\end{array}$ & Sí & Sí & Sí & Sí & Sí & Sí \\
\hline 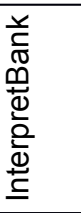 & Sí & A & $89 €$ & Ambos & 35 & No & $\begin{array}{c}\text { Android } \\
\text { Win } \\
\text { iOS }\end{array}$ & Sí & Sí & Sí & Sí & Sí & Sí \\
\hline 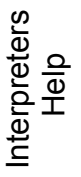 & Sí & $\underset{\text { (web) }}{\mathrm{A}}$ & $\begin{array}{c}19.99 € / \\
\text { month }\end{array}$ & Sin & Ilim. & No & $\begin{array}{c}\text { Android } \\
\text { Win } \\
\text { iOS }\end{array}$ & Sí & Sí & No & No & Sí & Sí \\
\hline $\begin{array}{l}\frac{0}{2} \\
\frac{y}{0} \\
0\end{array}$ & No & $\begin{array}{c}\mathrm{A} \\
\text { (web) }\end{array}$ & $\begin{array}{l}\text { On } \\
\text { request }\end{array}$ & Sin & $\Leftrightarrow$ & No & $\begin{array}{c}\text { Android } \\
\text { Win } \\
\text { iOS }\end{array}$ & Sí & No & No & Sí & Sí & Sí \\
\hline : & Sí & A & CHF 148 & Ambos & $\Leftrightarrow$ & No & Win & Sí & Sí & Sí & Sí & Sí & Sí \\
\hline
\end{tabular}

Tabla 1. Evaluación de las herramientas de gestión terminológica.

En los datos precedentes se observa que varias de las herramientas se ajustan mejor a las necesidades de la interpretación social que otras. Aunque algunas herramientas son populares entre los intérpretes, la realidad es que una de ellas, Lookup, no incluye a éstos entre sus potenciales usuarios.

Por lo que respecta a la disponibilidad, solo una de las herramientas analizadas, Glossary Assistant, es gratuita, el resto son de pago. 
Además, dos de las herramientas analizadas (Interpreter Help y Lookup) tienen un diseño basado en la web, así que no funcionan si el intérprete no dispone de conexión de internet. Por tanto, estas herramientas no son útiles para la interpretación social, puesto que el intérprete no sabe de antemano si va a disponer de conexión a Internet en el lugar de la interpretación.

Aunque cuatro de las herramientas (Glossary Assistant, Interplex UE, InterpreterBank e Interpreters Help) permiten escoger cualquier lengua, la realidad es que no permiten el empleo de sistemas de escritura diferentes como el ruso, el chino o el árabe.

Otra de las restricciones que hemos detectado es la relativa al sistema operativo. Una de las herramientas, Terminus, solo funciona con Windows, por lo que su utilización se restringe a determinadas tabletas y teléfonos móviles. Otra herramienta, Glossary Assistant, no está disponible para iOS, así que no pueden utilizarla los intérpretes sociales que trabajen con Apple.

Por otro lado, las interfaces de las herramientas analizadas son muy intuitivas, si bien el motor de búsqueda de Lookup es lento. Además, tres herramientas (Look Up, Glossary Assistant e Interpreter Help) no permiten filtrar los datos por campos.

Respecto al diseño de la base de datos, Interpreters Help no ofrece la opción de diseñar la estructura de la base, así que los intérpretes sociales no podrán crear campos que consideren relevantes para los proyectos de interpretación social.

No obstante, una característica positiva que es común a todas las herramientas analizadas se corresponde con la actualización de la base de datos, así que el intérprete social podrá modificar el contenido cuando lo precise. Además, todas las herramientas permiten también exportar e importar a otros formatos.

Para finalizar, los resultados obtenidos son alentadores y parcialmente satisfacen las necesidades de los intérpretes sociales cuando tienen que gestionar la terminología de una interpretación, sin embargo, todavía es necesario implementarlas.

\section{Conclusión}

Las herramientas de gestión terminológica son esenciales para los intérpretes sociales, puesto que contribuyen a almacenar, clasificar y recuperar el conocimiento especializado, así que permiten que su trabajo sea más eficiente. En este sentido, Stoll (2002) reconoce que la búsqueda terminológica con herramientas diseñadas para intérpretes libera la memoria a corto plazo y promueve la recuperación de más estructuras sintácticas dado que los conceptos se convierten en vocabulario casi activo para el intérprete.

Por tanto, en un futuro cercano, nuevas versiones de estas herramientas o incluso nuevas herramientas deberían cumplir con los requisitos que previamente hemos expuesto para satisfacer las necesidades de los intérpretes sociales: deberían ser asequibles, permitir su uso en diferentes dispositivos como teléfonos móviles, tabletas y portátiles, ser de fácil utilización, capaces de importar y exportar datos, dotadas de motores de búsqueda rápidos, que permitan diferentes posibilidades de búsqueda y diferentes sistemas de escritura. Además, los intérpretes sociales deberían ser capaces de definir la estructura de la base de datos y de poder actualizar los datos en cualquier momento para que la gestión terminoógica sea rápida, fiable y reutilizable en futuras interpretaciones.

Por otro lado, es necesario formar a los intérpretes, ya sean estudiantes o profesionales, tanto en su uso como en los procedimientos de búsqueda para que puedan optimizar las búsquedas y recuperar la información que necesitan en el menor tiempo posible.

Por último, entre nuestros planes de futuro destaca realizar un estudio empírico para conocer si las herramientas actuales satisfacen las necesidades de los intérpretes sociales. 
Los resultados que obtengamos podrán, a su vez, ser contrastados con las necesidades de otros tipos de intérpretes, como por ejemplo los intérpretes de conferencias.

\section{Referencias}

Bancroft, M. A. (2015). «Community interpreting. A Profession Rooted in Social Justice». En Mikkelson, H. y Jourdenais, R. (eds.). The Routledge Handbook of Interpreting. London/New York: Routledge, 217-235.

Bilgen, B. (2009). Investigating Terminology Management for Conference Interpreters. MA dissertation. Otawa: University of Otawa.

Costa, H., Corpas Pastor, G. y Durán Muñoz, I. (2014a). Technology-Assisted Interpreting. MultiLingual 143, 25(3), 27-32.

Costa, H., Corpas Pastor, G. y Durán Muñoz, I. (2014b). «A Comparative User Evaluation of Terminology Management Tools for Interpreters». In Proceedings of the 4th International Workshop on Computational Terminology (pp. 68-76) Dublin, Ireland, August 232014. Retrieved 13/05/2016 from http://www.aclweb.org/anthology/W14-4809.

Costa, H., Corpas Pastor, G. y Durán Muñoz, I. (2015). «An Interpreters' Guide to Selecting Terminology Management Tools». NATO Conference on Terminology Management. Brussels, Belgium. November 2015. https://eden.dei.uc.pt/ hpcosta/docs/papers/201511-NATO.pdf Fecha de consulta: 13.05.2016.

Gile, D. (1985). «Les termes techniques en interprétation simultanée». Meta: Translators' Journal, 30(3), 199-210.

Gile, D. (1986). «Le travail terminologique en interprétation de conférence». Multilingua 5(1), 31-36.

Gile, D. (1987). «La terminotique en interpretation de conférence: un potentiel à exploiter». Meta: Translators' Journal, 32(2), 164-169.

Hebenstreit, G., y Soukup-Unterweger, I. (2011). «Terminologiemanagement als Wissensmodellierung für das Community Interpreting». En Kainz, C., Prunc, E. y Schöger, R. (eds.). Modelling the Field of Community Interpeting. Questions of Methodology in Research and Training. Wien: LIT Verlag, 298-325.

ISO. (2014). 3611:2014(E) Interpreting - Guidelines for Community Interpreting. Geneva: International Standards Association.

Kutz, W. (2000). «Training für den Ernstfall. Warum und wie sich die Vorbereitung auf den Dolmetscheinsatz lohnt». MDÜ 3: 8-13.

Mikkelson, H. (2004). «The Professionalization of Community Interpreting». The AllC Webmagazine. http://aiic.net/page/1546/the-professionalization-of-communityinterpreting/lang/1 Fecha de consulta: 13.05.2016.

Moser-Mercer, B. (1992). «Banking on Terminology: Conference Interpreters in the Electronic Age». Meta: Translators' Journal, 37(3), 507-522.

Pöchhacker, F. (2011). «NT and Cl in IS: Taxonomies and Tensions in Interpreting Studies». En Blasco Mayor, M. J. y Jiménez Ivars, M. A. (eds.). Interpreting Naturally: A Tribute to Brian Harris. Bern: Peter Lang, 217-235.

Roberts, R. (1994). «Community Interpreting Today and Tomorrow». En Krawutschke, P. (Ed.). 35th Annual Conf. of the American Translators Association. Medford, NJ: Learned Information, 127-138. 
Rodríguez, N. \& Schnell, B. (2009). A Look at Terminology Adapted to the Requirements Interpretation. Language Update, 6(1), 21-27.

http://www.btb.termiumplus.gc.ca/tpv2guides/guides/favart/index-eng.html? lang=eng\&lettr=indx_autr8gijKBACeGnl\&page=9oHAHvmFzkgE.html Fecha de consulta: 27.07.2016.

Rütten, A. (2014a). Booth-friendly terminology management programs for interpreters - a market snapshot. http://blog.sprachmanagement.net/?p=265 Fecha de consulta: 27.07.2016.

Rütten, A. (2014b). Booth-friendly terminology management revisited - 2 newcomers. http://blog.sprachmanagement.net/?p=305 Fecha de consulta: 27.07.2016.

Shlesinger, M. (2011). An equal footing: On the role of community interpreters in multilingual societies.

http://www2.uef.fi/documents/1040001/1040008/Abstract_Miriam+Shlesinger.pdf/28a60d aa-d365-4991-a1d1-aa7cb5d9d26c Fecha de consulta: 27.07.2016.

Stoll, C. (2002). «Terminologiesysteme für Simultandolmetscher». MDÜ 3, 47-51.

Tipton, R. y Furmanek, O. (2016). Dialogue Interpreting. A Guide to Interpreting in Public Services and the Community. London/New York: Routledge.

Valero-Garcés, C. (2014). Communicating across Cultures. A Coursebook on Interpreting and Translating in Public Services and Institutions. Lanham/Plymouth: University Press of America.

\section{Páginas web}

Glossary Assistant: http://swiss32.com/ [Fecha de consulta: 27.07.2016].

Interplex: http://www.fourwillows.com/interplex.html [Fecha de consulta: 14.05.2016].

InterpretBank: www.interpretbank.de [Fecha de consulta: 14.05.2016].

Interpreters Help: https://interpretershelp.com/ [Fecha de consulta: 14.05.2016].

Lookup: http://www.dog-gmbh.de/software-products/lookup.html?L=1 [Fecha de consulta: 14.05.2016].

Terminus: http://www.wintringham.ch/cgi/ayawp.pl?T=terminus [Fecha de consulta: 14.05.2016]. 